PROCEEDINGS OF THE

AMERICAN MATHEMATICAL SOCIETY

Volume 127, Number 4, April 1999, Pages 1201-1208

S 0002-9939(99)04654-7

\title{
LIPSCHITZ PRECOMPACTNESS FOR CLOSED NEGATIVELY CURVED MANIFOLDS
}

\author{
IGOR BELEGRADEK
}

(Communicated by Christopher Croke)

\begin{abstract}
We prove that, given a integer $n \geq 3$ and a group $\pi$, the class of closed Riemannian $n$-manifolds of uniformly bounded negative sectional curvatures and with fundamental groups isomorphic to $\pi$ is precompact in the Lipschitz topology. In particular, the class breaks into finitely many diffeomorphism types.
\end{abstract}

\section{$\S 1$. INTRODUCTION}

According to the Mostow rigidity theorem, the isometry type of a closed locally symmetric negatively curved Riemannian manifold is uniquely determined by its fundamental group. This is no longer true for manifolds of variable sectional curvature. The purpose of this note is to observe that there are essentially finitely many possibilities for the geometry and topology of such manifolds provided the sectional curvature is pinched between two negative fixed constants.

1.1. Theorem. For any number $b \in[-1,0)$ and a group $\pi$, the class $\mathcal{M}_{n, b, \pi}$ of closed Riemannian manifolds of dimension $n \geq 3$ with sectional curvatures in $[-1, b]$ and fundamental groups isomorphic to $\pi$ is precompact in the Lipschitz topology.

Recall that the class of all compact Riemannian manifolds of a given dimension has the so-called Lipschitz topology, namely, two manifolds $M$ and $N$ are said to be $\epsilon$-close if there exists a diffeomorphism $f: M \rightarrow N$ such that both $f$ and $f^{-1}$ are $e^{\epsilon}$-Lipschitz. A class of manifolds is called precompact if for any positive $\epsilon$, every sequence of manifolds in the class has a subsequence whose members are mutually $\epsilon$-close. A landmark theorem of Gromov asserts the Lipschitz precompactness of the class of closed Riemannian manifolds of uniformly bounded diameters and sectional curvatures and with a uniform lower bound on the injectivity radii. Here is an immediate corollary of Lipschitz precompactness.

1.2. Corollary. For any number $b \in[-1,0)$, a positive integer $n$, and a group $\pi$, there exist positive numbers $D>d$ depending only on $b$ and $\pi$ such that any manifold from $\mathcal{M}_{n, b, \pi}$ has diameter in $[d, D]$. The same conclusion is true for volume and injectivity radius.

Received by the editors July 30, 1997.

1991 Mathematics Subject Classification. Primary 53C20, 53C23; Secondary 20F32, 57R55.

Key words and phrases. Lipschitz convergence, negatively curved manifold.

(C)1999 American Mathematical Society 
Note that the corollary fails if $b=0$. Indeed, given a closed negatively curved manifold $M$, the rescaled manifold $k \cdot M$ has sectional curvature within $[1,0)$ for large $k$, while the diameters are unbounded. Combining 1.2 with the GromovFukaya pinching theorem $[\mathrm{F}]$ we get

1.3. Corollary. Given a group $\pi$ and a positive integer $n$, there exists $\epsilon \in(0,1)$ such that any manifold from $\mathcal{M}_{n,-1+\epsilon, \pi}$ is diffeomorphic to a manifold of constant negative sectional curvature.

Topology of closed negatively curved manifolds seems to be encoded in the fundamental group. It is a very particular case of the Borel conjecture that any two closed homotopy equivalent negatively curved manifolds must be homeomorphic. However, in higher dimensions there are homeomorphic negatively curved manifolds that are not diffeomorphic [FJ1, 2]. In fact, Farrell and Jones proved that given a closed real hyperbolic manifold $N$ of dimension $n \geq 5$ and $\epsilon>0$, there is a finite cover $N_{\epsilon} \rightarrow N$ such that for any smooth homotopy $n$-sphere $\Sigma$ the connected sum $N_{\epsilon} \# \Sigma$ has a Riemannian metric of sectional curvature within $[-1,-1+\epsilon]$. Moreover, given a pair of nondiffeomorphic homotopy spheres $\Sigma_{1}$ and $\Sigma_{2}$, the manifolds $N_{\epsilon} \# \Sigma_{1}$ and $N_{\epsilon} \# \Sigma_{2}$ are not diffeomorphic. The following result is another standard application of Lipschitz precompactness.

1.4. Corollary. For any number $b \in[-1,0)$ and a group $\pi$ there exist at most finitely many nondiffeomorphic closed Riemannian manifolds with sectional curvatures in $[-1, b]$ and fundamental groups isomorphic to $\pi$.

In dimensions $\geq 5$ a much stronger statement is true. Namely, a deep theorem of Farrell and Jones implies that any manifold homotopy equivalent to a closed nonpositively curved manifold $M$ must be homeomorphic to $M$ [FJ3] Since a manifold of dimension $\geq 5$ can have only finitely many smooth structures [KS], every homotopy type contains finitely many nondiffeomorphic closed nonpositively curved manifolds of dimension $\geq 5$.

No results similar to this theorem of Farrell and Jones are available in dimension four because it is unknown in this dimension if the topological surgery works for the word-hyperbolic fundamental groups. In general, one expects most closed 4manifolds to have more than one smooth structure.

According to the (as yet unproved) Geometrization Conjecture of Thurston, any closed negatively curved 3-manifold should admit a metric of constant negative curvature. In particular, if the conjecture is true, any two homotopy equivalent closed negatively curved 3-manifolds must be diffeomorphic, due to the Mostow rigidity theorem. Notice that for closed Haken 3-manifolds with no curvature assumptions, homotopy equivalence implies diffeomorphism [W]. The same is true for any closed irreducible 3-manifold that is homotopy equivalent to a 3-manifold of constant negative curvature [G], [GMT].

We finally sketch the proof of Theorem 1.1. In the case of pinched negative sectional curvature, arguments based on the Margulis lemma provide a universal lower bound on the injectivity radius, so there is no collapse. This guarantees precompactness in the pointed Lipschitz topology where limit points may be noncompact manifolds. It turns out to be technically convenient to think of closed manifolds from $\mathcal{M}_{n, b, \pi}$ as Hadamard manifolds equipped with the isometric, free, cocompact actions of $\pi$. Using a method due to Bestvina and Paulin and a famous result of Rips, Bestvina and Feighn on small actions on real trees, one can show that these 
actions do not "diverge". This implies that whenever a sequence $M_{k} \in \mathcal{M}_{n, b, \pi}$ converges in the pointed Lipschitz topology to a manifold $M$, the group $\pi_{1}(M)$ contains a subgroup isomorphic to $\pi$. Counting cohomological dimension, one can deduce that $M$ is compact, and hence $M_{k}$ converges to $M$ in the (nonpointed) Lipschitz topology.

The author is grateful to Mladen Bestvina and Bernhard Leeb for helpful discussions on the subject of this paper and to Bill Goldman for friendly support.

\section{§2. TWO TYPES OF CONVERGENCE}

2.1. Equivariant pointed Lipschitz topology. Let $\Gamma_{k}$ be a discrete subgroup of the isometry group of a complete Riemannian manifold $X_{k}$ and $p_{k}$ be a point of $X_{k}$. The class of all such triples $\left\{\left(X_{k}, p_{k}, \Gamma_{k}\right)\right\}$ can be given the so-called equivariant pointed Lipschitz topology $[\mathrm{F}]$; when $\Gamma_{k}$ is trivial this reduces to the usual pointed Lipschitz topology. For convenience of the reader we give here some definitions borrowed from $[\mathrm{F}]$.

For a group $\Gamma$ acting on a pointed metric space $(X, p, d)$ the set $\{\gamma \in \Gamma$ : $d(p, \gamma(p))<r\}$ is denoted by $\Gamma(r)$. An open ball in $X$ of radius $r$ with center at $p$ is denoted by $B_{r}(p, X)$.

For $i=1,2$, let $\left(X_{i}, p_{i}\right)$ be a pointed complete metric space with the distance function $d_{i}$ and let $\Gamma_{i}$ be a discrete group of isometries of $X_{i}$. In addition, assume that $X_{i}$ is a $C^{\infty}$-manifold. Take any $\epsilon>0$.

Then a quadruple $\left(f_{1}, f_{2}, \phi_{1}, \phi_{2}\right)$ of maps $f_{i}: B_{1 / \epsilon}\left(p_{i}, X_{i}\right) \rightarrow B_{1 / \epsilon}\left(p_{3-i}, X_{3-i}\right)$ and $\phi_{i}: \Gamma_{i}(1 / 3 \epsilon) \rightarrow \Gamma_{3-i}$ is called an $\epsilon$-Lipschitz approximation between the triples $\left(X_{1}, p_{1}, \Gamma_{1}\right)$ and $\left(X_{2}, p_{2}, \Gamma_{2}\right)$ if the following seven conditions hold:

- $f_{i}$ is a diffeomorphism onto its image;

- for each $x_{i} \in B_{1 / 3 \epsilon}\left(p_{i}, X_{i}\right)$ and every $\gamma_{i} \in \Gamma_{i}(1 / 3 \epsilon), f_{i}\left(\gamma_{i}\left(x_{i}\right)\right)=\phi_{i}\left(\gamma_{i}\right)\left(f_{i}\left(x_{i}\right)\right)$;

- for every $x_{i}, x_{i}^{\prime} \in B_{1 / \epsilon}\left(p_{i}, X_{i}\right), e^{-\epsilon}<d_{3-i}\left(f_{i}\left(x_{i}\right), f_{i}\left(x_{i}^{\prime}\right)\right) / d_{i}\left(x_{i}, x_{i}^{\prime}\right)<e^{\epsilon}$;

- $f_{i}\left(B_{1 / \epsilon}\left(p_{i}, X_{i}\right)\right) \supset B_{(1 / \epsilon)-\epsilon}\left(p_{3-i}, X_{3-i}\right)$ and $\phi_{i}\left(\Gamma_{i}(1 / 3 \epsilon)\right) \supset \Gamma_{3-i}(1 / 3 \epsilon-\epsilon)$;

- $f_{i}\left(B_{(1 / \epsilon)-\epsilon}\left(p_{i}, X_{i}\right)\right) \supset B_{1 / \epsilon}\left(p_{3-i}, X_{3-i}\right)$ and $\phi_{i}\left(\Gamma_{i}(1 / 3 \epsilon-\epsilon)\right) \supset \Gamma_{3-i}(1 / 3 \epsilon)$;

- $\left.f_{3-i} \circ f_{i}\right|_{B_{(1 / \epsilon)-\epsilon}\left(p_{i}, X_{i}\right)}=\mathrm{id}$ and $\left.\phi_{3-i} \circ \phi_{i}\right|_{\Gamma_{i}(1 / 3 \epsilon-\epsilon)}=\mathrm{id}$;

- $d_{3-i}\left(f_{i}\left(p_{i}\right), p_{3-i}\right)<\epsilon$.

We say a sequence of triples $\left(X_{k}, p_{k}, \Gamma_{k}\right)$ converges to $(X, p, \Gamma)$ in the equivariant pointed Lipschitz topology if, for any $\epsilon>0$, there is $k(\epsilon)$ such that for all $k>k(\epsilon)$, there exists an $\epsilon$-Lipschitz approximation between $\left(X_{k}, p_{k}, \Gamma_{k}\right)$ and $(X, p, \Gamma)$.

Notice that if all the groups $\Gamma_{k}$ are trivial, then $\Gamma$ is trivial; in this case we say that that $\left(X_{k}, p_{k}\right)$ converges to $(X, p)$ in the pointed Lipschitz topology. Note that if $X_{k}$ is a complete Riemannian manifold for all $k$, then the space $X$ is necessarily a $C^{\infty}$-manifold with a complete $C^{1, \alpha}$-Riemannian metric [GW].

If $X_{k}$ and $X$ are compact, then $\left(X_{k}, p_{k}\right)$ converges to $(X, p)$ in the pointed Lipschitz topology if and only if, for any $\epsilon>0$ there is $k(\epsilon)$ such that for all $k>k(\epsilon)$, there exists a diffeomorphism $f: X_{k} \rightarrow X$ such that both $f$ and $f^{-1}$ are $e^{\epsilon}$-Lipschitz. In this case we just say that the sequence $X_{k}$ converges to $X$ in the Lipschitz topology.

2.2. Pointwise convergence topology. Suppose that, for some $p_{k} \in X_{k}$, the sequence $\left(X_{k}, p_{k}\right)$ converges to $(X, p)$ in the pointed Lipschitz topology.

We say that a sequence $x_{k} \in X_{k}$ converges to $x \in X$ if, for some $\epsilon$, the sequence of $\epsilon$-Lipschitz approximations $\left(f_{k}, g_{k}\right)$ between $\left(X_{k}, p_{k}\right)$ and $(X, p)$ has the property 
that $d\left(f_{k}\left(x_{k}\right), x\right)=d_{k}\left(x_{k}, g_{k}(x)\right) \rightarrow 0$ as $k \rightarrow \infty$. Trivial example: if $\left(X_{k}, p_{k}\right)$ converges to $(X, p)$ in the pointed Lipschitz topology, then $p_{k}$ converges to $p$.

Given a sequence of isometries $\gamma_{k} \in \operatorname{Isom}\left(X_{k}\right)$ we say that $\gamma_{k}$ converges, if for any $x_{k} \in X_{k}, \gamma_{k}\left(x_{k}\right)$ converges. The limiting transformation $\gamma$ of $X$ is necessarily an isometry.

We say that a sequence of actions $\left(X_{k}, p_{k}, \rho_{k}\right)$ converges to an action $(X, p, \rho)$ in the pointwise convergence topology if $\rho_{k}(\gamma)$ converges to $\rho(\gamma)$ for every $\gamma \in \pi$.

Clearly, if $\pi$ is generated by a finite set $S$, then in order to prove $\rho_{k} \rightarrow \rho$, it suffices to check that $\rho_{k}(\gamma)$ converges to $\rho(\gamma)$ for every $\gamma \in S$.

Let $d_{k}: X_{k} \times X_{k} \rightarrow \mathbb{R}$ be the distance function. It is easy to check that a sequence of actions $\left(X_{k}, p_{k}, \rho_{k}\right)$ is precompact in the pointwise convergence topology (i.e. every subsequence of $\left(X_{k}, p_{k}, \rho_{k}\right)$ has a converging subsequence) if for any $\gamma \in \pi$ and any (or, equivalently, some) $x_{k} \in X_{k}$, the sequence $d_{k}\left(x_{k}, \rho_{k}(\gamma)\left(x_{k}\right)\right)$ is bounded (the proof is in the spirit of [KN, 4.7]). Again, if $\pi$ is generated by a finite subset $S$ it is enough to verify the above for elements of $S$ only.

2.3. Motivating example. Let $X$ be a complete Riemannian manifold. Consider the isometry group $\operatorname{Isom}(X)$ of $X$ and let $\pi$ be a group.

The space $\operatorname{Hom}(\pi, \operatorname{Isom}(X))$ has a natural topology (which is usually called "algebraic topology" or "pointwise convergence topology"), namely $\rho_{k}$ is said to converge to $\rho$ if, for each $\gamma \in \pi, \rho_{k}(\gamma)$ converges to $\rho(\gamma)$ in the Lie group $\operatorname{Isom}(X)$. Note that if $\pi$ is finitely generated, this topology on $\operatorname{Hom}(\pi, \operatorname{Isom}(X))$ coincides with the compact-open topology.

Certainly, for any $p \in X$, the constant sequence $(X, p)$ converges to itself in pointed Lipschitz topology. Then, obviously, the sequence $\left(X, p, \rho_{k}\right)$ converges in the pointwise convergence topology (as defined in 2.2) if and only if $\rho_{k} \in$ $\operatorname{Hom}(\pi, \operatorname{Isom}(X))$ converges in the algebraic topology.

2.4. Lemma. Let $\rho_{k}: \pi \rightarrow \operatorname{Isom}\left(X_{k}\right)$ be a sequence of free, isometric actions of a discrete group $\pi$ on complete Riemannian $n$-manifolds $X_{k}$.

If the sequence $\left(X_{k}, p_{k}, \rho_{k}(\pi)\right)$ converges in the equivariant pointed Lipschitz topology to $(X, p, \Gamma)$ and $\left(X_{k}, p_{k}, \rho_{k}\right)$ converges to $(X, p, \rho)$ in the pointwise convergence topology, then

(1) $\Gamma$ acts freely, and

(2) $\rho$ is injective, and

(3) $\rho(\pi) \subset \Gamma$.

Proof. (1) Assume $\gamma \in \Gamma$ and $\gamma(x)=x$. Choose $\epsilon \in(0,1 / 10)$ so that there is an $\epsilon$-approximation $\left(f_{k}, g_{k}, \phi_{k}, \tau_{k}\right)$ of $\left(X_{k}, p_{k}, \rho_{k}\right)$ and $(X, p, \Gamma)$ and $x \in B(p, \epsilon / 10)$. Then $g_{k}(x)=g_{k}(\gamma(x))=\tau_{k}(\gamma)\left(g_{k}(x)\right)$. Since $\rho_{k}(\pi)$ acts freely, $\tau_{k}(\gamma)=\mathrm{id}$. By the same argument $\tau_{k}(\mathrm{id})=\mathrm{id}$. Hence id $=\phi_{k}\left(\tau_{k}(\mathrm{id})\right)=\phi_{k}(\mathrm{id})=\phi_{k}\left(\tau_{k}(\gamma)\right)=\gamma$ as desired.

(2) Assume $\rho(\gamma)=$ id. Fix any $\epsilon \in(0,1 / 10)$. Take $x \in B(p, \epsilon / 10)$ and consider an $\epsilon$-approximation $\left(f_{k}, g_{k}, \phi_{k}, \tau_{k}\right)$ of $\left(X_{k}, p_{k}, \rho_{k}\right)$ and $(X, p, \Gamma)$. We have $g_{k}(x) \rightarrow x$ and $\rho_{k}(\gamma)\left(g_{k}(x)\right) \rightarrow \rho(\gamma)(x)=x$. Note that $d\left(x, \phi_{k}\left(\rho_{k}(\gamma)\right)(x)\right)$ is equal to

$$
d\left(f_{k}\left(g_{k}(x)\right), f_{k}\left(\rho_{k}(\gamma)\left(g_{k}(x)\right)\right)\right)<e^{\epsilon} d_{k}\left(g_{k}(x), \rho_{k}(\gamma)\left(g_{k}(x)\right)\right) \underset{k \rightarrow \infty}{\longrightarrow}>0 .
$$

Therefore, $\phi_{k}\left(\rho_{k}(\gamma)\right)=$ id, because $\Gamma$ is a discrete subgroup that acts freely. Hence $\rho_{k}(\gamma)=\tau_{k}\left(\phi_{k}\left(\rho_{k}(\gamma)\right)\right)=\tau_{k}(\mathrm{id})=\mathrm{id}$. Since $\rho_{k}$ is injective, $\gamma=\mathrm{id}$ as claimed. 
(3) We need to show that $\rho(\gamma) \in \Gamma$, for any $\gamma \in \pi$. We can assume $\rho(\gamma) \neq$ id. Choose $\epsilon \in(0,1 / 10)$ so that the ball $B(p, 1 / 11 \epsilon)$ contains $\rho(\gamma)(p)$ and consider an $\epsilon$-approximation $\left(f_{k}, g_{k}, \phi_{k}, \tau_{k}\right)$ of $\left(X_{k}, p_{k}, \rho_{k}\right)$ and $(X, p, \Gamma)$.

Then for all large enough $k, \rho_{k}(\gamma) \in B\left(p_{k}, 1 / 10 \epsilon\right)$. Look at $\tau_{k}\left(\rho_{k}(\gamma)\right) \in \Gamma(1 / 9 \epsilon)$. Since the set $\Gamma(1 / 9 \epsilon)$ is finite, we can pass to a subsequence to assume that $\tau_{k}\left(\rho_{k}(\gamma)\right)$ is equal to $\gamma_{\epsilon} \in \Gamma(1 / 9 \epsilon)$; thus $\rho_{k}(\gamma)=\phi_{k}\left(\gamma_{\epsilon}\right)$.

Take an arbitrary $x \in B(p, 1 / 9 \epsilon)$. Then $g_{k}(x) \rightarrow x$ and, hence, $\rho_{k}(\gamma)\left(g_{k}(x)\right)$ converges to $\rho(\gamma)(x)$. Notice that $\rho_{k}(\gamma)\left(g_{k}(x)\right)=\phi_{k}\left(\gamma_{\epsilon}\right)\left(g_{k}(x)\right) \rightarrow \gamma_{\epsilon}(x)$. So $\rho(\gamma)(x)=\gamma_{\epsilon}(x)$ for any $x \in B(p, 1 / 9 \epsilon)$.

Thus, for any small enough $\epsilon$, we have found $\gamma_{\epsilon} \in \Gamma$ that is equal to $\rho(\gamma)$ on the ball $B(p, 1 / 9 \epsilon)$. Since $\Gamma$ acts freely, $\gamma_{\epsilon}=\gamma_{\epsilon^{\prime}}$ for all $\epsilon^{\prime} \leq \epsilon$, that is the element $\gamma_{\epsilon} \in \Gamma$ is independent of $\epsilon$. Thus $\rho(\gamma)=\gamma_{\epsilon}$ everywhere and hence $\rho(\gamma) \in \Gamma$.

\section{§3. MAIN LEMMA}

3.1. Proposition. Assume that $\pi$ is a finitely presented discrete group, that is not virtually nilpotent and does not have a nontrivial decomposition into an amalgamated product or an $H N N$-extension over a virtually nilpotent group.

Let $\rho_{k}: \pi \rightarrow \operatorname{Isom}\left(X_{k}\right)$ be an arbitrary sequence of free and isometric actions of $\pi$ on Hadamard $n$-manifolds $X_{k}$. Assume that the sectional curvatures of $X_{k}$ lie in $[-1, b]$ for $b<0$.

Then, for some $p_{k} \in X_{k},\left(X_{k}, p_{k}, \rho_{k}(\pi)\right)$ is precompact in the equivariant pointed Lipschitz topology and $\left(X_{k}, p_{k}, \rho_{k}\right)$ is precompact in the pointwise convergence topology.

Proof. Let $S \subset \pi$ be a finite subset that generates $\pi$. For $x \in X_{k}$, we denote by $D_{k}(x)$ the diameter of the set $\rho_{k}(S)(x)$. Set $D_{k}=\inf _{x \in X_{k}} D_{k}(x)$.

Suppose $D_{k}$ is unbounded. Then it follows from a work of Bestvina and Paulin [Bes], [P1], [P2] (cf. [KL]) that there exists an action of $\pi$ on a real tree with no global fixed point and virtually nilpotent arc stabilizers. For completeness we briefly review this construction. The rescaled pointed Hadamard manifold $\frac{1}{D_{k}} \cdot X_{k}$ has sectional curvature $\leq b \cdot D_{k} \rightarrow-\infty$ as $k \rightarrow \infty$. Find $q_{k} \in X_{k}$ such that $D_{k}\left(q_{k}\right) \leq D_{k}+1 / k$. Consider the sequence of triples $\left(\frac{1}{D_{k}} \cdot X_{k}, q_{k}, \rho_{k}\right)$. Repeating an argument of Paulin [P2, $\S 4]$, we can pass to a subsequence that converges to a triple $\left(X_{\infty}, q_{\infty}, \rho_{\infty}\right)$. (For the definition of the convergence see [P1], [P2]. Paulin calls it "convergence in the Gromov topology".)

The limit space $X_{\infty}$ is a length space of curvature $-\infty$, that is a real tree. Because of rescaling, the limit space has a natural isometric action $\rho_{\infty}$ of $\pi$. By the Margulis lemma the stabilizer of any non-degenerate segment is virtually nilpotent (cf. [P1]). One can check that the action $\rho_{\infty}$ has no global fixed point [P1], [P2]. Then it is a standard fact that there exists a unique $\pi$-invariant subtree $T$ of $X_{\infty}$ that has no proper $\pi$-invariant subtree. In fact $T$ is the union of all the axes of all hyperbolic elements in $\pi$.

Note that any increasing sequence of virtually nilpotent subgroups of $\pi$ is stationary. Indeed, since a virtually nilpotent group is amenable, the union $U$ of an increasing sequence $U_{1} \subset U_{2} \subset U_{3} \subset \ldots$ of virtually nilpotent subgroups is also an amenable group. If the fundamental group of a complete manifold of pinched negative curvature is amenable, it must be finitely generated [BS], [Bow]. In particular, $U$ is finitely generated; hence $U_{n}=U$ for some $n$. Thus, the $\pi$-action on the tree $T$ is stable [BF, Proposition 3.2(2)]. 
We summarize that the $\pi$-action on $T$ is stable, has virtually nilpotent arc stabilizers and no proper $\pi$-invariant subtree. Therefore, the Rips machine [BF, Theorem 9.5] produces a splitting of $\pi$ over a virtually solvable group. Any amenable subgroup of $\pi$ is virtually nilpotent [BS], [Bow]; hence $\pi$ splits over a virtually nilpotent group. This is a contradiction with the assumption that $D_{k}$ is unbounded.

Thus the sequence $D_{k}\left(q_{k}\right)$ is bounded. As we observed in 1.2 , the sequence $\left(X_{k}, q_{k}, \rho_{k}\right)$ is precompact in the pointwise convergence topology since the sequence $D_{k}\left(q_{k}\right)$ is bounded. Note that for any $p_{k} \in X_{k}$ such that $d_{k}\left(p_{k}, q_{k}\right)$ is bounded, the sequence $\left(X_{k}, p_{k}, \rho_{k}\right)$ is also precompact in the pointwise convergence topology.

We now find $p_{k} \in X_{k}$ such that $d_{k}\left(p_{k}, q_{k}\right)$ is bounded and $\left(X_{k}, p_{k}, \rho_{k}\right)$ is precompact in the equivariant pointed Lipschitz topology.

Let $\mu_{n}>0$ be the Margulis constant for dimension $n$. Set $r_{k}=D_{k}\left(q_{k}\right)+1$. Note that $r_{k}$ is a bounded sequence of positive numbers such that, for any $\gamma \in S$, $\rho_{k}(\gamma)\left(B\left(q_{k}, r_{k}\right)\right) \cap B\left(q_{k}, r_{k}\right) \neq \emptyset$.

Assume that for some $k$ and for every point $x$ of the $r_{k}$-ball centered at $q_{k}$, there exists $\gamma \in \pi$ such that $d_{k}\left(x, \rho_{k}(\gamma)(x)\right)<\mu_{n} / 2$. Then the whole ball $B\left(q_{k}, r_{k}\right)$ projects into the thin part $\left\{\operatorname{InjRad}<\mu_{n} / 2\right\}$ under the projection $\pi_{k}: X_{k} \rightarrow$ $X_{k} / \rho_{k}(\pi)$. Thus the ball $B\left(p, r_{k}\right)$ lies in a connected component $W$ of the $\pi_{k^{-}}$ preimage of the thin part of $X_{k} / \rho_{k}(\pi)$. According to [BGS, p. 111] the stabilizer of $W$ in $\rho_{k}(\pi)$ is virtually nilpotent and, moreover, the stabilizer contains every element $\gamma \in \rho_{k}(\pi)$ with $\gamma(W) \cap W \neq \emptyset$. Therefore, the whole group $\rho_{k}(\pi)$ stabilizes $W$. Hence $\rho_{k}(\pi)$ must be virtually nilpotent. As $\rho_{k}$ is injective, $\pi$ is virtually nilpotent. A contradiction.

Thus, for every $k$, there exists $p_{k} \in B\left(q_{k}, r_{k}\right)$ such that the injectivity radius of $X_{k} / \rho_{k}(\pi)$ at the point $\pi_{k}\left(p_{k}\right)$ is at least $\mu_{n} / 2$. Thus, by a theorem of Fukaya [F], $\left(X, p_{k}, \rho_{k}(\pi)\right)$ is precompact in the equivariant pointed Lipschitz topology.

3.2. Remark. It is well-known that the fundamental group of a closed negatively curved manifold of dimension $\geq 3$ is not virtually nilpotent $[\mathrm{Y}]$ and does not split over a virtually nilpotent group (the latter is an easy group cohomology exercise, see for example $[\mathrm{Bel}])$.

\section{$\S 4$. MAIN THEOREM}

4.1. Theorem. Let $\rho_{k}: \pi \rightarrow \operatorname{Isom}\left(X_{k}\right)$ be a sequence of free, isometric and cocompact actions of a discrete group $\pi$ on complete Riemannian $n$-manifolds $X_{k}$.

Assume that for some $p_{k} \in X_{k},\left(X_{k}, p_{k}, \rho_{k}(\pi)\right)$ converges in the equivariant pointed Lipschitz topology to $(X, p, \Gamma)$ and that $\left(X_{k}, p_{k}, \rho_{k}\right)$ converges in the pointwise convergence topology to $(X, p, \rho)$. Suppose that the manifolds $X_{k}$ and $X$ are contractible.

Then the sequence of manifolds $\left\{X_{k} / \rho_{k}(\pi)\right\}$ converges to $X / \Gamma$ in the Lipschitz topology.

Proof. Since $\rho(\pi) \subset \Gamma$, the quotient map $X \rightarrow X / \Gamma$ factors through $X / \rho(\pi)$. The fundamental group of $X / \rho(\pi)$ is isomorphic to $\pi$ because $X$ is contractible and $\rho$ is an isomorphism onto its image.

The manifolds $X_{k} / \rho_{k}(\pi)$ and $X / \rho(\pi)$ are aspherical and with fundamental groups isomorphic to $\pi$. Hence, $X_{k} / \rho_{k}(\pi)$ and $X / \rho(\pi)$ are homotopy equivalent. Since $X_{k} / \rho_{k}(\pi)$ is compact, so is $X / \rho(\pi)$ (look at $\mathbb{Z}_{2}$-top-dimensional homology). Therefore, $X / \Gamma$ is compact. Hence there exists a ball $B(p, r) \subset X$ that projects onto $X / \Gamma$. Take any $\epsilon$ so that $r<1 / 3 \epsilon$. 
Take $k$ so large that there is an $\epsilon$-Lipschitz approximation $\left(\tilde{f}_{k}, \tilde{g}_{k}, \phi_{k}, \tau_{k}\right)$ of $\left(X_{k}, p_{k}, \rho_{k}(\pi)\right)$ and $(X, p, \Gamma)$. The equivariant diffeomorphism $\tilde{g}_{k}: B(p, 1 / 3 \epsilon) \rightarrow$ $X_{k}$ descends to a smooth embedding $g_{k}$ of a closed manifold $X / \Gamma$ into the closed manifold $X_{k} / \Gamma_{k}$. Similarly, $\tilde{f}_{k}$ descends to a smooth embedding $f_{k}: X_{k} / \Gamma_{k} \rightarrow X / \Gamma$ which is the inverse of $g_{k}$. By construction $g_{k}$ and $f_{k}$ are $\epsilon$-Lipschitz. Therefore, $X_{k} / \Gamma_{k}$ converges to $X / \Gamma$ in the Lipschitz topology.

4.2. Corollary. Let $\rho_{k}: \pi \rightarrow \operatorname{Isom}\left(X_{k}\right)$ be a sequence of free, isometric and cocompact actions of a discrete group $\pi$ on Hadamard $n$-manifolds $X_{k}$.

Assume that for some $p_{k} \in X_{k},\left(X_{k}, p_{k}, \rho_{k}(\pi)\right)$ converges in the equivariant pointed Lipschitz topology to $(X, p, \Gamma)$ and that $\left(X_{k}, p_{k}, \rho_{k}\right)$ converges in the pointwise convergence topology to $(X, p, \rho)$.

Then the sequence of manifolds $\left\{X_{k} / \rho_{k}(\pi)\right\}$ converges to $X / \Gamma$ in the Lipschitz topology.

Proof. According to 4.1, it suffices to show that $X$ is contractible. Indeed, any spheroid in $X$ lies in the diffeomorphic image of a metric ball in $X_{j}$. Any metric ball in a Hadamard manifold is contractible. Thus $\pi_{*}(X)=1$ as desired.

4.3. Corollary. For any number $b \in[-1,0)$ and a group $\pi$, the class $\mathcal{M}_{n, b, \pi}$ of closed Riemannian manifolds of dimension $n \geq 3$ with sectional curvatures in $[-1, b]$ and fundamental groups isomorphic to $\pi$ is precompact in the Lipschitz topology.

Proof. Combine 3.1, 3.2 and 4.2.

\section{REFERENCES}

[BGS] W. Ballmann, M. Gromov, V. Schroeder, Manifolds of nonpositive curvature, Birkhauser, Progress in mathematics, vol. 61, 1985. MR 87h:53050

[Bel] I. Belegradek, Intersections in hyperbolic manifolds, Geometry \& Topology 2 (1998), 117-144, electronic: http://www.maths.warvick.ac.uk/gt/.

[Bes] M. Bestvina, Degenerations of the hyperbolic space, Duke Math. J 56 (1988), 143-161. MR 89m:57011

[BF] M. Bestvina and M. Feighn, Stable actions of groups on real trees, Invent. Math. 121 (2) (1995), 287-321. MR 96h:20056

[BS] M. Burger and V. Schroeder, Amenable groups and stabilizers of measures on the boundary of a Hadamard manifold, Math. Ann. 276 (3) (1987), 505-514. MR 88b:53049

[Bow] B. H. Bowditch, Discrete parabolic groups, J. Differential Geom. 38 (3) (1993), 559-583. MR 94h:53046

[FJ1] F. T. Farrell and L. E. Jones, Negatively curved manifolds with exotic smooth structures, J. Amer. Math. Soc. 2 (4) (1989), 899-908. MR 90f:53075

[FJ2] Complex hyperbolic manifolds and exotic smooth structures., Invent. Math. 117 (1) (1989), 57-74. MR 95e:57052

[FJ3] - Topological rigidity for compact non-positively curved manifolds; Proc. Sympos. Pure Math., 54, Part 3, Amer. Math. Soc., 1993, pp. 229-274. MR 94m:57067

[F] K. Fukaya, Theory of convergence for Riemannian orbifolds, Japan. J. Math. (N. S.) 12 (1) (1986), 121-160. MR 89e:53083

[G] D. Gabai, On the geometric and topological rigidity of hyperbolic 3-manifolds, J. Amer. Math. Soc. 10 (1) (1997), 37-74. MR 97h:57028

[GMT] D. Gabai, G. R. Meyerhoff, and N. Thurston, Homotopy Hyperbolic 3-Manifolds are Hyperbolic, MSRI Preprint No. 1996-058 (1996).

[GW] R. E. Greene and H. Wu, Lipschitz convergence of Riemannian manifolds, Pacific J. Math. 131 (1) (1988), 119-141. MR 89g:53063

[KL] M. Kapovich and B. Leeb, On asymptotic cones and quasi-isometry classes of fundamental groups of 3-manifolds, Geom. Funct. Anal. 5 (3) (1995), 582-603. MR 96e:57006 
[KS] R. S. Kirby and L. S. Siebenmann, Foundational essays on topological manifolds, smoothings, and triangulations, Annals of Mathematics Studies, No. 88. Princeton University Press, Princeton, 1977. MR 58:31082

[KN] S. Kobayashi and K. Nomidzu, Foundations of differential geometry, Vol. I, Interscience Publishers, a division of John Wiley \& Sons, 1963. MR 97c:53001a; MR 27:2945

[P1] F. Paulin, Topologie de Gromov équivariante, structures hyperboliques et arbres réels, Invent. Math. 94 (1) (1988), 53-80. MR 90d:57015

[P2] - Outer automorphisms of hyperbolic groups and small actions on $\mathbb{R}$-trees, Arboreal group theory, Math. Sci. Res. Inst. Publ., 19, Springer, 1991, pp. 331-343. MR 92g:57003

[W] F. Waldhausen, On irreducible 3-manifolds which are sufficiently large, Ann. Math. 87 (1968), 56-88.

[Y] S.-T. Yau, On the fundamental group of compact manifolds of nonpositive curvature, Ann. Math. 93 (2) (1971), 579-585. MR 44:956

Department of Mathematics, University of Maryland, College Park, Maryland 20742

E-mail address: igorb@math.umd.edu

Current address: Department of Mathematics and Statistics, McMaster University, 1280 Main

St. West, Hamilton, Ontario, Canada L8S 4K1

E-mail address: belegi@icarus.math.mcmaster.ca 